\title{
BANNAI-ITO POLYNOMIALS AND DRESSING CHAINS
}

\author{
MAXIM DEREVYAGIN, SATOSHI TSUJIMOTO, LUC VINET, AND ALEXEI ZHEDANOV \\ (Communicated by Sergei K. Suslov)
}

\begin{abstract}
Schur-Delsarte-Genin (SDG) maps and Bannai-Ito polynomials are studied. SDG maps are related to dressing chains determined by quadratic algebras. The Bannai-Ito polynomials and their kernel polynomials the complementary Bannai-Ito polynomials - are shown to arise in the framework of the SDG maps.
\end{abstract}

\section{INTRODUCTION}

It is well known that periodic dressing chains for Schrödinger operators can be used to characterize finite-gap potentials [27]. There were also some attempts to adapt the scheme for tridiagonal operators and orthogonal polynomials [15], 21], 20]. Naturally, this research leads to the following question: what families of orthogonal polynomials can be described via periodic dressing chains for tridiagonal operators?

In this paper we pursue the exploration of this question and study the interplay between dressing chains for tridiagonal matrices and known families of orthogonal polynomials. More precisely, the goal of the present paper is twofold. First, we propose a way to define Darboux transformations for bilinear pencils of the form

$$
A-\lambda B-x I,
$$

where $A, B$ are tridiagonal semi-infinite matrices and $I$ is the identity operator. One shall see that these transformations lead to dressing chains related to quadratic algebras. Remarkably, the pencils $A-\lambda B-x I$ appear in different contexts [3, 4], 13] (see also [7], 23], and [26], where similar objects arise). Second, we construct as an explicit example the dressing chain related to the Bannai-Ito polynomials [2], 25]. Its presentation in an open form will require setting the Bannai-Ito polynomials in the framework of Schur-Delsarte-Genin maps.

The paper is organized as follows. In Section 2 we define the generic Darboux transformations for tridiagonal matrices and identify the relation between 1-periodic dressing chains and quadratic algebras. Examples of dressing chains generated by orthogonal polynomials on the unit circle are given in Section 3. The next section

Received by the editors January 8, 2013.

2010 Mathematics Subject Classification. Primary 42C05; Secondary 17B80, 33C45, 47B36.

Key words and phrases. Darboux transformations, dressing chains, orthogonal polynomials, Schur-Delsarte-Genin map, Bannai-Ito polynomials, quadratic algebras.

The first author acknowledges the support of the European Research Council under the European Union Seventh Programme (FP/2007-2013)/ERC grant agreement No. 259173. The first and fourth authors thank the University of Montreal for its hospitality in the course of this study.

The research of the third author was supported in part by a research grant from the Natural Sciences and Engineering Research Council (NSERC) of Canada. 
is concerned with Schur-Delsarte-Genin maps, which are essentially the bridges between dressing chains and orthogonal polynomials on the unit circle. The rest of the paper deals with the Bannai-Ito polynomials as an explicit example. Namely, in Section 5 we transform the complementary Bannai-Ito polynomials into polynomials on the unit circle. These new polynomials turn out to be related to symmetrized Racah-Wilson polynomials, as shown in Section 6. Finally, in Section 7 we discuss the role of the Bannai-Ito polynomials in Schur-Delsarte-Genin maps.

\section{Generic Darboux transformations}

In this section we give the definition of the generic Darboux transformations and identify quadratic algebras of tridiagonal operators as dressing chains.

Before giving the general abstract idea, let us briefly recall the interconnection between discrete Darboux transformations and dynamical systems. Consider a finite dimensional case and let $J$ be a finite Jacobi matrix; that is,

$$
J=\left(\begin{array}{cccc}
a_{0} & b_{0} & & \\
b_{0} & a_{1} & \ddots & \\
& \ddots & \ddots & b_{N-1} \\
& & b_{N-1} & a_{N}
\end{array}\right),
$$

where $a_{j} \in \mathbb{R}$ and $b_{j}>0$. In addition, assume that $J$ is positive definite. Then $J$ admits the Cholesky decomposition

$$
J=L L^{*}
$$

where $L$ is a lower triangular matrix and $\operatorname{det} L \neq 0$. One can see that in this case $L$ is bidiagonal. Recall [5], 36] that the Darboux transformation of $J$ is defined as follows:

$$
J=L L^{*} \mapsto \tilde{J}=L^{*} L .
$$

Clearly, $\tilde{J}$ is also a Jacobi matrix and the spectrum $\sigma(\tilde{J})$ of $\tilde{J}$ coincides with the spectrum $\sigma(J)$ of $J$. Indeed, from the definition we get that

$$
\tilde{J} L^{*}=L^{*} J
$$

Thus $(\tilde{J}-\lambda I) L^{*}=L^{*}(J-\lambda I)$, where $\lambda$ is a complex number and $I$ is the corresponding identity operator. Hence, $\sigma(\tilde{J})=\sigma(J)$ since $\operatorname{det} L \neq 0$. Furthermore, the eigenvectors $\tilde{f}_{j}$ and $f_{j}$ of $\tilde{J}$ and $J$, respectively, are related as follows:

$$
\tilde{f}_{j}=L^{*} f_{j}
$$

It follows that the iterates of the Darboux transformation applied to $J$ are isospectral and related to an isospectral flow [32.

Now we are in a position to give a more formal definition of the Darboux transformation of tridagonal semi-infinite matrices:

$$
J=\left(\begin{array}{cccc}
\alpha_{0} & \beta_{0} & & \\
\gamma_{0} & \alpha_{1} & \beta_{1} & \\
& \gamma_{1} & \alpha_{2} & \beta_{2} \\
& & \gamma_{2} & \ddots
\end{array}\right), \quad\left|\beta_{j}\right|+\left|\gamma_{j}\right| \neq 0, \quad j \in \mathbb{Z}_{+},
$$

which are the discrete analog of the Schrödinger operator and appear in the theory of orthogonal polynomials and continued fractions [18, 22]. 
Definition 2.1. Let $J$ be of the form (2.1) and let $\psi$ be a column vector defined by the condition

$$
J \psi=0 .
$$

Also, let $D$ be a semi-infinite nonzero matrix such that the product $D \psi$ makes sense. Introduce a new vector

$$
\tilde{\psi}=D \psi
$$

Now, suppose that there exist semi-infinite matrices $K, \tilde{J}$ that satisfy the intertwining relation

$$
\tilde{J} D=K J .
$$

Then the operator $\tilde{J}$ is called the Darboux transformation of $J$ and it is easy to see that

$$
\tilde{J} \tilde{\psi}=0 .
$$

We will call this scheme the generic Darboux scheme, and formula (2.3) describes the Darboux transformation from the vector $\psi$ to the vector $\tilde{\psi}$. Formula (2.4) is an algebraic relation between the operators $J$ and $\tilde{J}$.

Obviously, the generic Darboux scheme gives a family of Darboux transformations depending on the choice of $D$. For example, for any two Jacobi matrices $J$ and $\tilde{J}$ one can trivially take $D=c J$ and $K=c \tilde{J}$, where $c$ is a nonzero complex number. Therefore, any Jacobi matrix $\tilde{J}$ can be considered as the generic Darboux transformation of the given matrix $J$. Clearly, this is not too helpful and generally leads to trivial results. However, there are many nontrivial explicit examples which can be obtained by putting some requirements on $D, K$ and by letting the matrices $J, \tilde{J}$ depend on parameters. Let us consider the simplest such case,

$$
J(\lambda)=A-\lambda I,
$$

where $\lambda$ is a parameter and $A$ is a monic Jacobi matrix with real entries that do not depend on $\lambda$. In this case, the entries of $\psi$ are the corresponding orthogonal polynomials evaluated at $\lambda$. Let us fix $\lambda$ and suppose that $J(\lambda)$ admits the $L U$ decomposition, that is, $J(\lambda)=L U$, where $L$ and $U$ are lower and upper triangular matrices. Then introducing $\tilde{J}(\lambda)=\tilde{A}-\lambda I=U L$ we see that (2.4) is satisfied for $K=D=U$. Besides, the entries of $\tilde{\psi}=D \psi$ are related to the orthogonal polynomials corresponding to $\tilde{A}$ [5], 36] (see also [10] for some nonclassical cases).

Another example can be obtained by considering the following form of the matrices $J$ and $\tilde{J}$ :

$$
J(\lambda)=A-\lambda B, \quad \tilde{J}(\lambda)=\tilde{A}-\lambda \tilde{B},
$$

where $A$ and $B$ are some tridiagonal matrices. Assuming that the operators $D$ and $K$ do not depend on $\lambda$, we obtain that (2.4) is equivalent to the following condition [34, 37]:

$$
\tilde{A} D=K A, \quad \tilde{B} D=K B .
$$

Actually, this entails the generalized eigenvalue problem

$$
A \psi=\lambda B \psi \text {. }
$$

Note that such problems appear in mechanics [14], in the theory of biorthogonal rational functions [37, and in interpolation problems [12. In this case, the Darboux 
transformation (2.3) gives a new eigenvector $\tilde{\psi}$ of the new generalized eigenvalue problem

$$
\tilde{A} \tilde{\psi}=\lambda \tilde{B} \tilde{\psi} .
$$

Finally, we are ready to discuss the generalization that is of interest in this paper. Namely, consider the following form of $J$ :

$$
J(\lambda, x)=A-\lambda B-x I,
$$

where the tridiagonal matrices $A$ and $B$ are independent of the real parameters $\lambda$ and $x$. We see that when one fixes $\lambda$ the problem for $J(\lambda, x)$ reduces to (2.6) and $J(\lambda, x)$ becomes (2.7) if $x$ is fixed.

Note that the initial condition for $\psi$,

$$
(A-\lambda B-x) \psi=0,
$$

is a typical 2-parameter problem [1], [19]. Our purpose is to describe 1-periodic dressing chains 27] related to such problems. Before giving the precise definition, let us specify the definition of the Darboux transformation of $J$ of the form (2.11). In this case, the Darboux transformation is obtained from the generic Darboux scheme (see Definition 2.1), where we want the intertwining (Darboux) operators $D$ and $K$ to have the forms

$$
D=r_{1} A+r_{2} B+r_{0} I, \quad K=s_{1} A+s_{2} B+s_{0} I,
$$

where $r_{i}, s_{i}$ are some real numbers. As always, this definition can lead to nontrivial results if the matrix $D$ is not proportional to $J$.

Definition 2.2. Let $J$ be a tridiagonal matrix given by (2.11). We say that $J$ generates a 1-periodic dressing chain if the Darboux transformation (2.4) subject to the Ansatz (2.13) gives a tridiagonal matrix of the form

$$
\tilde{J}=\widetilde{J(\lambda, x)}=A-\tilde{\lambda} B-\tilde{x} I,
$$

where $\tilde{x}, \tilde{\lambda}$ are some numbers which can be considered as the transformation of $x$ and $\lambda$. Notice that $\tilde{J}$ generates a new 2 -parameter problem

$$
(A-\tilde{\lambda} B-\tilde{x}) \tilde{\psi}=0
$$

similar to (2.12) but with transformed parameters $\tilde{\lambda}$ and $\tilde{x}$.

In other words, it means that the Darboux transformation applied to $J(\lambda, x)$ belongs to the linear span generated by $A, B$, and $I$. The following statement gives a description of the 1-periodic dressing chains related to $J(\lambda, x)$.

Theorem 2.3. Let the matrix $J$ be given by (2.11). If $J$ generates a 1-periodic dressing chain, then the quadratic relation

$$
\xi_{1} A^{2}+\xi_{2} B^{2}+\xi_{3} A B+\xi_{4} B A+\eta_{1} A+\eta_{2} B+\zeta I=0
$$

has a solution in $\xi_{i}, \eta_{i}$, and $\zeta$. This dressing chain is nontrivial if the condition

$$
\left|\xi_{1}\right|+\left|\xi_{2}\right|+\left|\xi_{3}\right|+\left|\xi_{4}\right|+\left|\eta_{1}\right|+\left|\eta_{2}\right|+|\zeta| \neq 0
$$

is satisfied for the solution.

Proof. The proof is immediate by making the substitution (2.11), (2.14) and (2.13) to (2.4). 
Remark 2.4. It should be mentioned that martingale orthogonal polynomials satisfy (2.12) 3. Furthermore, the quadratic algebraic relations (2.16) were considered in [3] and [4] in connection with quadratic harnesses and martingale orthogonal polynomials. Actually, it was shown that the Jacobi matrices corresponding to the Askey-Wilson polynomials satisfy (2.16). Moreover, the quadratic algebra (2.16) is related to an exactly solvable box-and-ball kinetic model in physics [13].

Note that the algebra (2.16) remains the same under the generic affine transformations $A \rightarrow \alpha_{1} A+\alpha_{2} B+\alpha_{0}, B \rightarrow \beta_{1} A+\beta_{2} B+\beta_{0}$. Using this observation we can put the quadratic algebra (2.16) in canonical forms. In fact, such a program was realized for self-adjoint operators in [17. We are not going to do this in the present paper. Here we shall give a particular example of the quadratic algebra (2.16) that is related to Schur-Delsarte-Genin maps [11] and not covered in [3] and [4]. One of the main goals of this paper is hence to show that the Jacobi matrices corresponding to the big -1 Jacobi polynomials and the Bannai-Ito polynomials satisfy the relation (2.16), thereby extending the class of polynomials related to quadratic algebras.

\section{Orthogonal polynomials on the Unit CIRCle AND QUADRATIC ALGEBRAS}

In this section we introduce a 2-parameter pencil and show that it is related to quadratic algebras and therefore to 1-periodic dressing chains.

Let us start by recalling that monic orthogonal polynomials $\Phi_{n}(z)=z^{n}+$ $O\left(z^{n-1}\right)$ on the unit circle satisfy the recurrence relation

$$
\Phi_{n+1}(z)=z \Phi_{n}(z)-\bar{a}_{n} \Phi_{n}^{*}(z)
$$

with the initial condition $\Phi_{0}(z)=1$; here the polynomials $\Phi_{n}^{*}(z)$ are defined as follows:

$$
\Phi_{n}^{*}(z)=z^{n} \overline{\Phi_{n}(1 / \bar{z})}
$$

(see [18). The recurrence coefficients $a_{n}=-\overline{\Phi_{n+1}(0)}$ are called reflection parameters (sometimes also referred to as the Schur, Geronimus, Verblunsky, ... parameters). It follows that $\left|a_{n}\right|<1$ for $n=0,1,2, \ldots$ Conversely (see [18]), polynomials satisfying (3.1) with this condition on $a_{n}$ are orthogonal.

In what follows we will consider only the case where the $a_{n}$ are real.

For convenience, we always assume that

$$
a_{-1}=-1 \text {. }
$$

The dual recurrence relation is

$$
\Phi_{n+1}^{*}(z)=\Phi_{n}^{*}(z)-a_{n} z \Phi_{n}(z) .
$$

It is standard to introduce the parameters

$$
r_{n}=\sqrt{1-a_{n}^{2}}
$$

(in our case, the square root is assumed to be positive). 
Let us introduce the block-diagonal matrices

$$
L=\left(\begin{array}{ccccccc}
a_{0} & r_{0} & & & & & \\
r_{0} & -a_{0} & & & & & \\
& & a_{2} & r_{2} & & & \\
& & r_{2} & -a_{2} & & & \\
& & & & a_{4} & r_{4} & \\
& & & & r_{4} & -a_{4} & \\
& & & & & & \ddots
\end{array}\right)
$$

and

$$
M=\left(\begin{array}{cccccccc}
1 & & & & & & & \\
& a_{1} & r_{1} & & & & & \\
& r_{1} & -a_{1} & & & & & \\
& & & a_{3} & r_{3} & & & \\
& & & r_{3} & -a_{3} & & & \\
& & & & a_{5} & r_{5} & \\
& & & & r_{5} & -a_{5} & \\
& & & & & & & \ddots
\end{array}\right) .
$$

Both $L$ and $M$ are block-diagonal unitary matrices. Define also the 5-diagonal unitary matrix $U[33$, 18]:

$$
U=L M
$$

(which is called the "Zigzag matrix" in [33] and the "CMV matrix" in [18]).

Next, consider a linear pencil of matrices,

$$
K(\lambda)=L+\lambda M,
$$

with an arbitrary parameter $\lambda$. So, the matrix $K(\lambda)$ is a nondegenerate symmetric tridiagonal matrix

$$
K(\lambda)=\left(\begin{array}{ccccc}
a_{0}+\lambda & r_{0} & & & \\
r_{0} & -a_{0}+\lambda a_{1} & \lambda r_{1} & & \\
& \lambda r_{1} & a_{2}-\lambda a_{1} & r_{2} & \\
& & r_{2} & -a_{2}+\lambda a_{3} & \lambda r_{3} \\
& & & & \ddots
\end{array}\right) .
$$

Here, nondegenerate means that all off-diagonal entries of the matrix $K(\lambda)$ are nonzero if $\lambda \neq 0$ and $\left|a_{i}\right| \neq 1$.

Hence one can define a family of formal monic orthogonal polynomials $Q_{n}(x ; \lambda)$ depending on the argument $x$ and an additional parameter $\lambda$. These polynomials are uniquely defined through the 3 -term recurrence relation

$$
Q_{n+1}(x ; \lambda)+b_{n}(\lambda) Q_{n}(x ; \lambda)+u_{n}(\lambda) Q_{n-1}(x ; \lambda)=x Q_{n}(x ; \lambda),
$$

where

$$
b_{n}(\lambda)=\left\{\begin{array}{cc}
a_{n}-\lambda a_{n-1} & \text { if } n \text { is even, } \\
\lambda a_{n}-a_{n-1} & \text { if } n \text { is odd }
\end{array}\right.
$$

and

$$
u_{n}(\lambda)=\left\{\begin{array}{c}
\lambda^{2}\left(1-a_{n-1}^{2}\right) \text { if } n \text { is even } \\
1-a_{n-1}^{2} \text { if } n \text { is odd. }
\end{array}\right.
$$


Note that the eigenvalue problem for the orthogonal polynomials $Q_{n}(x ; \lambda)$ can be presented in algebraic form as

$$
(L+\lambda M-x I) \vec{q}=0,
$$

where $\vec{q}$ is a vector constructed from the (nonmonic) polynomials $Q_{n}(x ; \lambda)$. Equation (3.11) contains two parameters $\lambda$ and $x$ and belongs to the class of the so-called multi-parameter eigenvalue problems [1, 19].

Theorem 3.1. Let $J=J(\lambda, x)=L+\lambda M-x I$. Then there exists a nontrivial choice of parameters $\tilde{x}$ and $\tilde{\lambda}$ such that the Darboux transformation of $J$ subject to (2.13) is of the form

$$
\tilde{J}=\widetilde{J(\lambda, x)}=L+\tilde{\lambda} M-\tilde{x} I .
$$

Thus, $J$ gives an explicit example of nontrivial 1-periodic dressing chains.

Proof. For this special case, relation (2.16) reduces to the following:

$$
\begin{gathered}
\tilde{\lambda} r_{1}=s_{2}, \quad r_{2}=\lambda s_{1}, \quad \lambda s_{0}-x s_{2}=\tilde{\lambda} r_{0}-x r_{2}, \quad r_{0}-\tilde{x} r_{1}=s_{0}-x s_{1}, \\
r_{1}+\tilde{\lambda} r_{2}-\tilde{x} r_{0}=s_{1}+\lambda s_{2}-x s_{0} .
\end{gathered}
$$

Putting $s_{1}=r_{1}=1$ we arrive at

$$
\tilde{x}^{2} \lambda-\tilde{x}(x \lambda+x \tilde{\lambda})+\lambda x^{2}=0,
$$

which is solvable. Moreover, it has a nontrivial solution (i.e. it is different from the trivial solution $\tilde{x}=x, \tilde{\lambda}=\lambda$ ).

Remark 3.2. Notice that

$$
J(\lambda, 0) J(-\lambda, 0)-q J(-\lambda, 0) J(\lambda, 0)=2\left(1-|\lambda|^{2}\right) I
$$

for $q=-1$. It is worth mentioning that some examples of the pencils of Jacobi matrices verifying (3.12) for $q \in(-1,1)$ were given in [4] and $[3$.

Remark 3.3. Interestingly, (3.12) becomes an anti-commutation relation at the same time that the spectrum of $K(\lambda)=L+\lambda M$ becomes an interval (the spectrum consists of two disjoint intervals for $|\lambda| \neq 1$ ) [11].

Taking into account Theorem 3.1, we see that the Jacobi matrices corresponding to the little and big -1 Jacobi polynomials [29, 30], 31] give explicit examples of dressing chains. The rest of the paper is devoted to constructing a realization related to the Bannai-Ito polynomials.

Remark 3.4. The main property of $J(\lambda, x)$ we used in the proof of Theorem 3.1 is the following:

$$
L^{2}=M^{2}=I .
$$

This property is based on the fact that $\left|a_{j}\right| \leq 1$. However, it is easy to see how to modify the matrices $L$ and $M$ in order to preserve the above property for an 
arbitrary real sequence $a_{j}$. Indeed, let us introduce the block-diagonal matrix

$$
L=\left(\begin{array}{ccccccc}
a_{0} & r_{0} & & & & & \\
\epsilon_{0} r_{0} & -a_{0} & & & & & \\
& & a_{2} & r_{2} & & & \\
& & \epsilon_{2} r_{2} & -a_{2} & & & \\
& & & & a_{4} & r_{4} & \\
& & & & \epsilon_{4} r_{4} & -a_{4} & \\
& & & & & & \ddots
\end{array}\right)
$$

and the block diagonal matrix

$$
M=\left(\begin{array}{cccccccc}
1 & & & & & & & \\
& a_{1} & r_{1} & & & & & \\
& \epsilon_{1} r_{1} & -a_{1} & & & & & \\
& & & a_{3} & r_{3} & & & \\
& & \epsilon_{3} r_{3} & -a_{3} & & & \\
& & & & a_{5} & r_{5} & \\
& & & & \epsilon_{5} r_{5} & -a_{5} & \\
& & & & & & \ddots
\end{array}\right),
$$

where $r_{j}=\sqrt{\left|1-a_{j}^{2}\right|}, \epsilon_{j}=-1$ if $\left|a_{j}\right|>1$, and $\epsilon_{j}=1$ if $\left|a_{j}\right| \leq 1$. Obviously, these new matrices $L$ and $M$ satisfy the main property: $L^{2}=M^{2}=I$. Furthermore, formulas (3.8), (3.9), and (3.10) remain the same. However, in the general case, the matrix $K(\lambda)=L+\lambda M$ is not symmetric in a Hilbert space but is rather symmetric in a Krein space (for example, see [9], [10]).

Finally, note that the Szegö orthogonal polynomials (satisfying (3.1)) with the condition $\left|a_{j}\right|>1$ were constructed in [28. Thus, those polynomials are also related to dressing chains.

\section{SChur-Delsarte-Genin MAPS}

In this section we give the precise definition of the Schur-Delsarte-Genin maps [11.

In order to make (3.8) more uniform, we will need the following special case of the Christoffel formula (see 24]).

Lemma 4.1. Let $P_{n}(x)$ be monic orthogonal polynomials satisfying the recurrence relation

$$
P_{n+1}(x)+\left(\theta-A_{n}-C_{n}\right) P_{n}(x)+C_{n} A_{n-1} P_{n-1}(x)=x P_{n}(x),
$$

with the standard initial conditions

$$
P_{0}=1, P_{1}(x)=x-\theta+A_{0} .
$$

Assume that the real coefficients $A_{n}, C_{n}$ are such that $A_{n-1}>0, C_{n}>0, n=$ $1,2 \ldots$, and that $C_{0}=0$. In this case, for any real $\theta$ one has that $P_{n}(\theta) \neq 0$ for $n=1,2, \ldots$. Therefore, one can define the new polynomials

$$
\tilde{P}_{n}(x)=\frac{P_{n+1}(x)-A_{n} P_{n}(x)}{x-\theta} .
$$


Then the monic polynomials $\tilde{P}_{n}(x)$ are orthogonal and satisfy the recurrence relation

$$
\tilde{P}_{n+1}(x)+\left(\theta-A_{n}-C_{n+1}\right) \tilde{P}_{n}(x)+C_{n} A_{n} \tilde{P}_{n-1}(x)=x \tilde{P}_{n}(x) .
$$

The inverse transformation from the polynomials $\tilde{P}_{n}(x)$ to the polynomials $P_{n}(x)$ is given by the formula

$$
P_{n}(x)=\tilde{P}_{n}(x)-C_{n} \tilde{P}_{n-1}(x) .
$$

To prove Lemma 4.1] it is sufficient to observe that relation 4.1) provides the explicit $L U$-factorization of the monic Jacobi matrix $J-\theta$ corresponding to the polynomials $P_{n}(x)$. The rest is an obvious reformulation of the theory of spectral (or Darboux) transformations [36, [5].

Note also that

$$
A_{n}=\frac{P_{n+1}(\theta)}{P_{n}(\theta)},
$$

which can easily be verified directly from (4.1).

Remark 4.2. If the polynomials $P_{n}(x)$ are orthogonal with respect to a weight function $w(x)$, then the polynomials $\tilde{P}_{n}(x)$ are orthogonal with respect to the weight function $(x-\theta) w(x)$.

$$
A_{n}=\left\{\begin{array}{c}
1-a_{n} \text { if } n \text { is even, } \\
\lambda\left(1-a_{n}\right) \text { if } n \text { is odd, }
\end{array} \quad C_{n}=\left\{\begin{array}{c}
\lambda\left(1+a_{n-1}\right) \text { if } n \text { is even, } \\
1+a_{n-1} \text { if } n \text { is odd. }
\end{array}\right.\right.
$$

Then the transformed polynomials $\tilde{Q}_{n}(x ; \lambda)$ satisfy the recurrence relation

$$
\tilde{Q}_{n+1}(x ; \lambda)+(-1)^{n}(\lambda-1) \tilde{Q}_{n}(x ; \lambda)+\lambda u_{n}^{*} \tilde{Q}_{n-1}(x ; \lambda)=x \tilde{Q}_{n}(x ; \lambda),
$$

where $u_{n}^{*}=\left(1+a_{n-1}\right)\left(1-a_{n}\right)$ does not depend on $\lambda$.

It will sometimes be useful to renormalize the polynomials $\tilde{Q}_{n}(x ; \lambda)$ as follows.

Lemma 4.3. Let the polynomials $\tilde{Q}_{n}(x ; \lambda)$ satisfy (4.8) and be orthogonal with respect to a weight function $w_{\lambda}(x)$ on the set $E_{\lambda}$ for positive $\lambda$. Then the monic polynomials defined via

$$
S_{n}\left(x ; \lambda, \lambda_{0}\right)=(\sqrt{\lambda})^{-n} \tilde{Q}_{n}\left(\sqrt{\lambda} x ; \lambda \lambda_{0}\right)
$$

are orthogonal with respect to the weight function $w_{\lambda}(\sqrt{\lambda} x)$ on the set $E_{\lambda}^{*}=\{x$ : $\left.\sqrt{\lambda} x \in E_{\lambda}\right\}$ and satisfy

$$
S_{n+1}\left(x ; \lambda, \lambda_{0}\right)+(-1)^{n} \chi S_{n}\left(x ; \lambda, \lambda_{0}\right)+\lambda_{0} u_{n}^{*} S_{n-1}\left(x ; \lambda, \lambda_{0}\right)=x S_{n}\left(x ; \lambda, \lambda_{0}\right),
$$

where $\chi=\lambda_{0} \sqrt{\lambda}-\frac{1}{\sqrt{\lambda}}$.

The converse is also true.

The proof is straightforward by making the substitutions $\lambda \rightarrow \lambda \lambda_{0}$ and $x \rightarrow \sqrt{\lambda} x$ in (4.8).

The above statement is the final step in the construction of the SDG map. The map from the polynomials $\Phi_{n}$ orthogonal on the unit circle to the polynomials $S_{n}$ defined by (4.10) is called the Schur-Delsarte-Genin map. Note that this map reduces to the Delsarte-Genin map [8] when $\lambda=\lambda_{0}=1$ (see [11, 35] for more details). 
To complete this section, we provide a lemma which will be useful in the identification of polynomial systems with known families of orthogonal polynomials.

Lemma 4.4 ([6]). Let $P_{n}(x)$ and $\tilde{P}_{n}(x)$ be two systems of orthogonal polynomials defined as in the previous lemma. Also, assume that the polynomials $P_{n}(x)$ are orthogonal with respect to a weight function $w(x)$ on the finite interval $[\alpha, \beta]$ and take any $\theta \in(-\infty, \alpha)$. Finally, define the monic polynomials

$$
S_{2 n}(x)=P_{n}\left(x^{2}+\alpha-c^{2}\right), \quad S_{2 n+1}(x)=(x-\chi) \tilde{P}_{n}\left(x^{2}+\alpha-c^{2}\right),
$$

where $c$ is a positive number such that $\sqrt{\alpha-\theta} \leq c$ and $\chi$ is a real number defined by the relation $\theta=\chi^{2}+\alpha-c^{2}$. Then the polynomials $S_{n}(x)$ are orthogonal with respect to the weight function

$$
(\operatorname{sign}(x))(x+\chi) w\left(x^{2}+\alpha-c^{2}\right)
$$

on the union of two intervals $\left[-\sqrt{\beta-\alpha+c^{2}},-c\right] \cup\left[c, \sqrt{\beta-\alpha+c^{2}}\right]$ and satisfy the recurrence relation

$$
S_{n+1}+(-1)^{n} \chi S_{n}(x)+v_{n} S_{n-1}(x)=x S_{n}(x),
$$

where $v_{n}$ are given by

$$
v_{2 n}=-C_{n}, v_{2 n+1}=-A_{n} .
$$

The converse statement is also true.

The orthogonality part of this lemma can be checked by straightforward computations. Substituting (4.11) into the corresponding three-term recurrence relations, we arrive at (4.13) and (4.14). For more details, see [6].

\section{Complementary Bannai-Ito polynomials}

In this section we show that the complementary Bannai-Ito polynomials can be obtained from the Schur-Delsarte-Genin transformation of some polynomials.

For the reader's convenience we begin with the explicit formulas for the general Bannai-Ito polynomials [2]:

$$
P_{n+1}(x)+\left(\rho_{1}-A_{n}-C_{n}\right) P_{n}(x)+A_{n-1} C_{n} P_{n-1}(x)=x P_{n}(x),
$$

where

$$
A_{n}=\left\{\begin{array}{ccc}
\frac{\left(n+1+2 \rho_{1}-2 r_{1}\right)\left(n+1+2 \rho_{1}-2 r_{2}\right)}{4\left(n+1-r_{1}-r_{2}+\rho_{1}+\rho_{2}\right)}, & n & \text { even, } \\
\frac{\left(n+1-2 r_{1}-2 r_{2}+2 \rho_{1}+2 \rho_{2}\right)\left(n+1+2 \rho_{1}+2 \rho_{2}\right)}{4\left(n+1-r_{1}-r_{2}+\rho_{1}+\rho_{2}\right)}, & n & \text { odd }
\end{array}\right.
$$

and

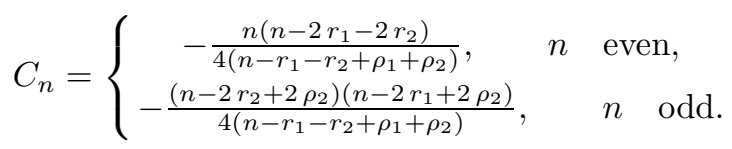

We see that the Bannai-Ito polynomials admit the representation (4.1) from Lemma 4.1. So, it is possible to introduce their companion polynomials [25, which we denote by $W_{n}(x)$, as follows:

$$
W_{n}(x)=\frac{P_{n+1}(x)-A_{n} P_{n}(x)}{x-\rho_{1}},
$$


with $A_{n}$ given by (5.2). The polynomials $W_{n}(x)$ satisfy the recurrence relation (4.4). In our special case, this relation reads

$$
W_{n+1}(x)+(-1)^{n} \rho_{2} W_{n}(x)+v_{n} W_{n-1}(x)=x W_{n}(x),
$$

where the coefficients $v_{n}$ have the following explicit form:

$$
\begin{aligned}
v_{2 n} & =-\frac{n\left(n+\rho_{1}-r_{1}+1 / 2\right)\left(n+\rho_{1}-r_{2}+1 / 2\right)\left(n-r_{1}-r_{2}\right)}{(2 n+1+g)(2 n+g)}, \\
v_{2 n+1} & =-\frac{(n+g+1)\left(n+\rho_{1}+\rho_{2}+1\right)\left(n+\rho_{2}-r_{1}+1 / 2\right)\left(n+\rho_{2}-r_{2}+1 / 2\right)}{(2 n+1+g)(2 n+g+2)},
\end{aligned}
$$

with $g=\rho_{1}+\rho_{2}-r_{1}-r_{2}$.

One can observe that the companion polynomials $W_{n}$ defined by (5.5) and the polynomials $S_{n}$ defined by (4.10) are, in fact, of the same nature. So, we can therefore try to identify them. First, let us write the equality

$$
v_{n}=\lambda_{0} u_{n}^{*}=\lambda_{0}\left(1+a_{n-1}\right)\left(1-a_{n}\right),
$$

for $n=0,1,2, \ldots$ From (5.7) we see that the parameters $a_{n}$ can be determined as

$$
a_{n}=1-\frac{v_{n}}{\lambda_{0}\left(1+a_{n-1}\right)},
$$

and $a_{0}, \lambda_{0}$ are arbitrary parameters. Setting

$$
a_{0}=1-2 \frac{\left(\rho_{1}-r_{2}+1 / 2\right)\left(-r_{1}-r_{2}\right)}{\left(-r_{2}-\rho_{2}-1 / 2\right)(g+1)}, \quad \lambda_{0}=\frac{1}{4}\left(-\rho_{2}-r_{2}-1 / 2\right)\left(\rho_{2}-r_{2}-1 / 2\right),
$$

we arrive at

$$
a_{n}=\left\{\begin{array}{ccc}
1-2 \frac{\left(\rho_{1}-r_{2}+(n+1) / 2\right)\left(-r_{1}-r_{2}+n / 2\right)}{\left(-\rho_{2}-r_{2}-1 / 2\right)(n+g+1)}, & n \text { even, } \\
1-2 \frac{(g+(n+1) / 2)\left(\rho_{2}-r_{2}+n / 2\right)}{\left(\rho_{2}-r_{2}-1 / 2\right)(n+g+1)}, & n & \text { odd. }
\end{array}\right.
$$

To complete the identification, it remains to take

$$
\lambda=\lambda_{B I}=\frac{4}{\left(-\rho_{2}-r_{2}-1 / 2\right)^{2}}
$$

to ensure that

$$
\lambda_{0} \sqrt{\lambda_{B I}}-\frac{1}{\sqrt{\lambda_{B I}}}=\rho_{2} .
$$

Remark 5.1. First, notice that

$$
\begin{aligned}
K^{2}\left(\lambda \lambda_{0}\right) & =\left(L+\lambda \lambda_{0} M\right)\left(L+\lambda \lambda_{0} M\right)=\left(1-\lambda \lambda_{0}\right)^{2} I+\lambda(L+M)^{2} \\
& =\frac{1}{\lambda}\left[\left(\lambda_{0} \sqrt{\lambda}-\frac{1}{\sqrt{\lambda}}\right)^{2} I+\lambda_{0}(L+M)^{2}\right] \\
& =\frac{1}{\lambda}\left[\chi^{2} I+\lambda_{0}(L+M)^{2}\right], \quad \lambda \neq 0 .
\end{aligned}
$$

From (5.11) and the spectral mapping theorem we see that $\sigma(K(\cdot))$ consists of numbers that are the square root of a quadratic polynomial in $\lambda$. Since it is known that the orthogonality measure for the Bannai-Ito polynomials (and, thus, for the complementary Bannai-Ito polynomials) has a uniform grid of mass points 25, one can conclude that, for the point $\lambda=\lambda_{0} \lambda_{B I}$ and the coefficients $a_{n}$ defined in (5.9), the quadratic polynomial $\chi^{2}+\lambda_{0} \mu_{n}^{2}$, where $\mu_{n}$ is an eigenvalue of $L+M$, becomes an exact square. 


\section{RaCAh-Wilson POlynomials on the Unit CiRCle}

We see from (5.11) that in order to characterize the spectrum of $K(\lambda)$ we need to know that spectrum precisely, at one point $\lambda$ at least. In this section, we identify such a determining point and show that this point is the preimage of the SDG map corresponding to the symmetrized Racah-Wilson polynomials.

Recall [16] that the Wilson polynomials $\tilde{W}_{n}(x)$ are defined through the 3-term recurrence relation

$$
\tilde{W}_{n+1}(x)+\left(A_{n}+C_{n}-\beta_{1}^{2}\right) \tilde{W}_{n}(x)+A_{n-1} C_{n} \tilde{W}_{n-1}(x)=x \tilde{W}_{n}(x),
$$

where $\sigma=\beta_{1}+\beta_{2}+\beta_{3}+\beta_{4}$ and the coefficients $A_{n}, C_{n}$ are as follows:

$$
\begin{gathered}
A_{n}=\frac{(n+\sigma-1)\left(n+\beta_{1}+\beta_{2}\right)\left(n+\beta_{1}+\beta_{3}\right)\left(n+\beta_{1}+\beta_{4}\right)}{(2 n+\sigma-1)(2 n+\sigma)}, \\
C_{n}=\frac{n\left(n+\beta_{2}+\beta_{3}-1\right)\left(n+\beta_{2}+\beta_{4}-1\right)\left(n+\beta_{3}+\beta_{4}-1\right)}{(2 n+\sigma-1)(2 n+\sigma-2)} .
\end{gathered}
$$

We shall refer to the above polynomials as the Racah-Wilson polynomials since for the discrete measure case (which is essential in the study of the Bannai-Ito polynomials) they represent the same type of polynomials.

Regarding the aim of this section, let us first note that it was already shown in 25] that the complementary Bannai-Ito polynomials can be constructed from the Racah-Wilson polynomials. Namely, the complementary Bannai-Ito polynomials are obtained from the Racah-Wilson polynomials by applying the Chihara construction (in other words, by using Lemma 4.4).

We can repeat this construction for our purpose, but it is in fact enough to observe that setting $\lambda=1 / \lambda_{0}$ at the last stage of the identification in the previous section leads to the following symmetric polynomials:

$$
S_{n+1}\left(x ; \lambda_{0}\right)+v_{n} S_{n-1}\left(x ; \lambda_{0}\right)=x S_{n}\left(x ; \lambda_{0}\right) .
$$

Clearly, the polynomials $i^{n} S_{n}\left(i x ; \lambda_{0}\right)$ are symmetrized Racah-Wilson polynomials. More precisely, the polynomials $i^{n} S_{n}\left(i x ; \lambda_{0}\right)$ can be obtained from $\tilde{W}_{n}(x)$ by using Lemma 4.4 with $\chi=0$ as desired. Obviously, we have

$$
\beta_{1}=\rho_{2}, \quad \beta_{2}=1+\rho_{1}, \quad \beta_{3}=-r_{1}+\frac{1}{2}, \quad \beta_{4}=-r_{2}+\frac{1}{2} .
$$

It should be stressed that the choice $\chi=0$ (that is, $\lambda \lambda_{0}=1$, and the corresponding Jacobi matrix being $K(1)=L+M$ ) corresponds to the classical Delsarte-Genin map. Therefore, the orthogonal polynomials associated with the reflection parameters

$$
a_{n}=\left\{\begin{array}{ccc}
1-2 \frac{\left(\beta_{2}+\beta_{4}+n / 2-1\right)\left(\beta_{3}+\beta_{4}+n / 2-1\right)}{\left(\beta_{4}-\beta_{1}-1\right)(n+\sigma-1)}, & n & \text { even }, \\
1-2 \frac{(\sigma+n / 2-3 / 2)\left(\beta_{1}+\beta_{4}+n / 2-1 / 2\right)}{\left(\beta_{1}+\beta_{4}-1\right)(n+\sigma-1)}, & n & \text { odd }
\end{array}\right.
$$

are the preimages of the symmetrized Racah-Wilson polynomials under the DelsarteGenin map. 


\section{Bannai-Ito Polynomials}

In this section we show how the Bannai-Ito polynomials appear in the framework of the Schur-Delsarte-Genin maps.

First, notice that the complementary Bannai-Ito polynomials are obtained from the Bannai-Ito polynomials through a Christoffel transformation. Actually, the same can be said about the polynomials $S_{n}$ and $Q_{n}$ defined by (4.10) and (3.8), respectively. Since we have already identified $S_{n}$ with the complementary Bannai-Ito polynomials, one could expect that there is a similar relation between the BannaiIto polynomials and the polynomials $Q_{n}$. However, it is easy to see from (4.7), (5.2), (5.3), and (5.9) that the polynomials $Q_{n}$ cannot be identified with the Bannai-Ito polynomials. Generally speaking, the reason is that they are related by a Uvarov transformation and do not coincide. Nevertheless, the Uvarov transformation can be realized via the following substitution:

$$
\rho_{1} \mapsto-r_{2}-\frac{1}{2}, \quad \rho_{2} \mapsto \rho_{1}, \quad r_{1} \mapsto r_{1}, \quad r_{2} \mapsto-\rho_{2}-\frac{1}{2} .
$$

Indeed, applying (7.1) to (5.9) gives

$$
a_{n}=\left\{\begin{array}{ccc}
1-2 \frac{\left(\rho_{1}-r_{2}+(n+1) / 2\right)\left(\rho_{1}-r_{1}+(n+1) / 2\right)}{\left(\rho_{1}-\rho_{2}\right)(n+g+1)}, & n & \text { even, } \\
1-2 \frac{(g+(n+1) / 2)\left(\rho_{1}+\rho_{2}+(n+1) / 2\right)}{\left(\rho_{1}+\rho_{2}\right)(n+g+1)}, & n & \text { odd. }
\end{array}\right.
$$

Next, setting

$$
\lambda=\lambda_{0} \lambda_{B I}=\frac{\rho_{2}+\rho_{1}}{\rho_{2}-\rho_{1}}
$$

the calculation of $b_{n}(\lambda)$ and $u_{n}(\lambda)$ (see formulas (3.9) and (3.10) for these new coefficients $a_{n}$ leads to the equalities

$$
b_{n}(\lambda)=\frac{2}{\rho_{2}-\rho_{1}}\left(\rho_{1}-A_{n}-C_{n}\right), \quad u_{n}(\lambda)=\frac{4}{\left(\rho_{2}-\rho_{1}\right)^{2}} A_{n-1} C_{n},
$$

where $A_{n}$ and $C_{n}$ are defined by (5.2) and (5.3), respectively.

According to Lemma 4.3 and (7.3), we may see that the polynomials $Q_{n}(x ; \lambda)$ and the Bannai-Ito polynomials (5.1) coincide up to a renormalization.

Remark 7.1. To explain the choice of $\lambda=\lambda_{0} \lambda_{B I}$, notice that the complementary Bannai-Ito polynomials are the Christoffel transforms of the Bannai-Ito polynomials and that this $\lambda$ was picked for the SDG map to give the complementary Bannai-Ito polynomials. Thus, if we want to get the Bannai-Ito polynomials as the polynomials $Q_{n}$, the same $\lambda$ has to be taken under the transformation (7.1).

It is also natural from the point of view of the mass points of the orthogonality measure. Indeed, the sets of mass points for the Bannai-Ito polynomials and the complementary Bannai-Ito polynomials should be the same except possibly for one point.

Finally, let us summarize the results on the Bannai-Ito polynomials.

Theorem 7.2. The following statements hold true:

(1) The complementary Bannai-Ito polynomials are the SDG images of the Szegö polynomials corresponding to the parameters (5.9).

(2) The Bannai-Ito polynomials coincide with the polynomials $Q_{n}$ defined by (3.8), and in this case the parameters $a_{j}$ are given by (17.2).

(3) The difference between (5.9) and (7.2) is the transformation (7.1). 


\section{REFERENCES}

[1] F. V. Atkinson, Multiparameter eigenvalue problems. Volume I: Matrices and compact operators, Mathematics in Science and Engineering, Vol. 82, Academic Press, New York, 1972. MR.0451001 (56 \#9291)

[2] Eiichi Bannai and Tatsuro Ito, Algebraic combinatorics. I. Association schemes, The Benjamin/Cummings Publishing Co., Inc., Menlo Park, CA, 1984. MR882540 (87m:05001)

[3] Włodzimierz Bryc, Wojciech Matysiak, and Jacek Wesołowski, Quadratic harnesses, qcommutations, and orthogonal martingale polynomials, Trans. Amer. Math. Soc. 359 (2007), no. 11, 5449-5483, DOI 10.1090/S0002-9947-07-04194-3. MR2327037(2008j:60176)

[4] Włodek Bryc and Jacek Wesołowski, Askey-Wilson polynomials, quadratic harnesses and martingales, Ann. Probab. 38 (2010), no. 3, 1221-1262, DOI 10.1214/09-AOP503. MR2674998(2011d:60226)

[5] M. I. Bueno and F. Marcellán, Darboux transformation and perturbation of linear functionals, Linear Algebra Appl. 384 (2004), 215-242, DOI 10.1016/j.laa.2004.02.004. MR2055354 (2005b:15027)

[6] T. S. Chihara, On kernel polynomials and related systems, Boll. Un. Mat. Ital. (3) 19 (1964), 451-459. MR0176121(31 \#396)

[7] S. Corteel, R. Stanley, D. Stanton, and L. Williams, Formulae for Askey-Wilson moments and enumeration of staircase tableaux, Trans. Amer. Math. Soc. 364 (2012), no. 11, 60096037, DOI 10.1090/S0002-9947-2012-05588-7. MR2946941

[8] Philippe Delsarte and Yves V. Genin, The split Levinson algorithm, IEEE Trans. Acoust. Speech Signal Process. 34 (1986), no. 3, 470-478, DOI 10.1109/TASSP.1986.1164830. MR844658 (87f:94007)

[9] Maxim Derevyagin, Generalized Jacobi operators in Krein spaces, J. Math. Anal. Appl. 349 (2009), no. 2, 568-582, DOI 10.1016/j.jmaa.2008.09.032. MR2456213(2010d:47054)

[10] Maxim Derevyagin and Vladimir Derkach, Darboux transformations of Jacobi matrices and Padé approximation, Linear Algebra Appl. 435 (2011), no. 12, 3056-3084, DOI 10.1016/j.laa.2011.05.035. MR2831597 (2012g:47089)

[11] Maxim Derevyagin, Luc Vinet, and Alexei Zhedanov, CMV matrices and little and big -1 Jacobi polynomials, Constr. Approx. 36 (2012), no. 3, 513-535, DOI 10.1007/s00365-0129164-0. MR.2996442

[12] Maxim S. Derevyagin and Alexei S. Zhedanov, An operator approach to multipoint Padé approximations, J. Approx. Theory 157 (2009), no. 1, 70-88, DOI 10.1016/j.jat.2008.07.002. MR2500154(2010c:41012)

[13] Fabian H. L. Essler and Vladimir Rittenberg, Representations of the quadratic algebra and partially asymmetric diffusion with open boundaries, J. Phys. A 29 (1996), no. 13, 3375-3407, DOI 10.1088/0305-4470/29/13/013. MR.1400161 (97b:82017)

[14] Graham M. L. Gladwell, Inverse problems in vibration, 2nd ed., Solid Mechanics and its Applications, vol. 119, Kluwer Academic Publishers, Dordrecht, 2004. MR2102477 (2005g:74059)

[15] F. Alberto Grünbaum and Luc Haine, Orthogonal polynomials satisfying differential equations: the role of the Darboux transformation, Symmetries and integrability of difference equations (Estérel, PQ, 1994), CRM Proc. Lecture Notes, vol. 9, Amer. Math. Soc., Providence, RI, 1996, pp. 143-154. MR1416834 (97m:33003)

[16] Roelof Koekoek, Peter A. Lesky, and René F. Swarttouw, Hypergeometric orthogonal polynomials and their q-analogues. With a foreword by Tom H. Koornwinder. Springer Monographs in Mathematics, Springer-Verlag, Berlin, 2010. MR 2656096 (2011e:33029)

[17] Vasyl L. Ostrovskyı̌ and Yuriǔ S. Samoı̌lenko, On pairs of selfadjoint operators, Sem. Sophus Lie 3 (1993), no. 2, 185-218. MR1270175 (95b:47025)

[18] B. Simon, Orthogonal polynomials on the unit circle, American Mathematical Society Colloquium Publications, 54, Parts 1 and 2, American Mathematical Society, Providence, RI, 2005. MR2105088, MR2105089

[19] B. D. Sleeman, Multiparameter spectral theory in Hilbert space, J. Math. Anal. Appl. 65 (1978), no. 3, 511-530, DOI 10.1016/0022-247X(78)90160-9. MR510467 (80c:47021) 
[20] Vyacheslav Spiridonov, Luc Vinet, and Alexei Zhedanov, Spectral transformations, selfsimilar reductions and orthogonal polynomials, J. Phys. A 30 (1997), no. 21, 7621-7637, DOI 10.1088/0305-4470/30/21/030. MR.1603364

[21] Vyacheslav Spiridonov and Alexei Zhedanov, Discrete Darboux transformations, the discretetime Toda lattice, and the Askey-Wilson polynomials, Methods Appl. Anal. 2 (1995), no. 4, 369-398. MR1376302 (97f:33030)

[22] Vyacheslav Spiridonov and Alexei Zhedanov, Discrete-time Volterra chain and classical orthogonal polynomials, J. Phys. A 30 (1997), no. 24, 8727-8737, DOI 10.1088/03054470/30/24/031. MR1619538 (98m:39049)

[23] Wim Schoutens and Jozef L. Teugels, Lévy processes, polynomials and martingales. Special issue in honor of Marcel F. Neuts. Comm. Statist. Stochastic Models 14 (1998), no. 1-2, 335-349, DOI 10.1080/15326349808807475. MR1617536(99k:60118)

[24] Gábor Szegő, Orthogonal polynomials, 4th ed., American Mathematical Society, Colloquium Publications, Vol. XXIII, American Mathematical Society, Providence, R.I., 1975. MR0372517(51 \#8724)

[25] Satoshi Tsujimoto, Luc Vinet, and Alexei Zhedanov, Dunkl shift operators and BannaiIto polynomials, Adv. Math. 229 (2012), no. 4, 2123-2158, DOI 10.1016/j.aim.2011.12.020. MR2880217

[26] Masaru Uchiyama, Tomohiro Sasamoto, and Miki Wadati, Asymmetric simple exclusion process with open boundaries and Askey-Wilson polynomials, J. Phys. A 37 (2004), no. 18, 4985-5002, DOI 10.1088/0305-4470/37/18/006. MR2065218(2006d:82047)

[27] A. P. Veselov and A. B. Shabat, A dressing chain and the spectral theory of the Schrödinger operator (Russian, with Russian summary), Funktsional. Anal. i Prilozhen. 27 (1993), no. 2, 1-21, 96, DOI 10.1007/BF01085979; English transl., Funct. Anal. Appl. 27 (1993), no. 2, 81-96. MR 1251164 (94m:58179)

[28] Luc Vinet and Alexei Zhedanov, Szegö polynomials on the real axis, Integral Transform. Spec. Funct. 8 (1999), no. 1-2, 149-164, DOI 10.1080/10652469908819223. MR.1730600 (2000h:33010)

[29] Luc Vinet and Alexei Zhedanov, A 'missing' family of classical orthogonal polynomials, J. Phys. A 44 (2011), no. 8, 085201, 16, DOI 10.1088/1751-8113/44/8/085201. MR.2770369 (2012c:42065)

[30] Luc Vinet and Alexei Zhedanov, A limit $q=-1$ for the big $q$-Jacobi polynomials, Trans. Amer. Math. Soc. 364 (2012), no. 10, 5491-5507, DOI 10.1090/S0002-9947-2012-05539-5. MR2931336

[31] Luc Vinet and Alexei Zhedanov, A Bochner theorem for Dunkl polynomials, SIGMA Symmetry Integrability Geom. Methods Appl. 7 (2011), Paper 020, 9, DOI 10.3842/ SIGMA.2011.020. MR2804576 (2012f:33014)

[32] David S. Watkins, Isospectral flows, SIAM Rev. 26 (1984), no. 3, 379-391, DOI 10.1137/ 1026075. MR750456 (86d:58054)

[33] David S. Watkins, Some perspectives on the eigenvalue problem, SIAM Rev. 35 (1993), no. 3, 430-471, DOI 10.1137/1035090. MR1234638(94h:65039)

[34] D. S. Watkins and L. Elsner, Self-equivalent flows associated with the generalized eigenvalue problem, Linear Algebra Appl. 118 (1989), 107-127, DOI 10.1016/0024-3795(89)905764. MR995370 (90g:65056)

[35] Alexei Zhedanov, On some classes of polynomials orthogonal on arcs of the unit circle connected with symmetric orthogonal polynomials on an interval, J. Approx. Theory 94 (1998), no. 1, 73-106, DOI 10.1006/jath.1998.3179. MR.1637803(2000a:42040)

[36] Alexei Zhedanov, Rational spectral transformations and orthogonal polynomials, J. Comput. Appl. Math. 85 (1997), no. 1, 67-86, DOI 10.1016/S0377-0427(97)00130-1. MR1482157 (98h:42026)

[37] Alexei Zhedanov, Biorthogonal rational functions and the generalized eigenvalue problem, J. Approx. Theory 101 (1999), no. 2, 303-329, DOI 10.1006/jath.1999.3339. MR.1726460 (2000i:33014) 
Department of Mathematics MA 4-2, Technische Universität Berlin, Strasse des 17. Juni 136, D-10623 Berlin, Germany

Current address: Department of Mathematics, KU Leuven, Celestijnenlaan 200B, Box 2400, BE-3001 Leuven, Belgium

Department of Applied Mathematics and Physics, Graduate School of Informatics, Kyoto University, SAKyo-KU, KYOto 606-8501, JAPAN

Centre de recherches mathématiques, Université de Montréal, P.O. Box 6128, Centre-ville Station, Montréal, Québec, H3C 3J7, Canada

Donetsk Institute for Physics And Technology, 83114 Donetsk, Ukraine 